\title{
Ultrasonic Attenuation in the Vortex State of d-wave Superconductors
}

\author{
Tribikram Gupta ${ }^{1}$ and D. M. Gaitonde ${ }^{2}$ \\ ${ }^{1}$ Harish-Chandra Research Institute, \\ Chhatnag Road, Jhusi, Allahabad 211019, India \\ ${ }^{2}$ High Pressure Physics Division, Bhabha Atomic Research Centre, Mumbai 400085, India
}

\begin{abstract}
We calculate the low temperature quasi-particle contribution to the ultrasonic attenuation rate in the mixed state of d-wave superconductors. Our calculation is performed within the semi-classical approximation using quasi-particle energies that are Doppler shifted, with respect to their values in the Meissner phase, by the supercurrent associated with the vortices. We find that the attenuation at low temperatures and at fields $H_{c 1} \leq H \ll H_{c 2}$ has a temperature independent contribution which is proportional to $\sqrt{ } H$ where $H$ is the applied magnetic field. We indicate how our result in combination with the zero-field result for ultrasonic attenuation can be used to calculate one of the parameters $v_{F}, H_{c 2}$ or $\xi$ given the values for any two of them.
\end{abstract}

PACS numbers: 74.72.-h; 74.60.-w 
The discovery of high temperature superconductivity 1 in the cuprates has led to an intense theoretical effort at understanding the origins of the novel phenomena seen in these materials. The normal state of the cuprates is highly anomalous 2 and remains a puzzle that is still not understood. However, there is evidence 3 that well defined quasi-particles exist in the superconducting state. The superconducting state is now known 6 . whose order parameter has $d_{x^{2}-y^{2}}$ symmetry. The order parameter thus has nodes along the lines $k_{x}= \pm k_{y}$ in the two-dimensional Brillouin zone.

While the origin of the pairing interaction that leads to the occurence of d-wave superconductivity is not known at the present time, a great deal of progress in understanding the superconducting state can be made by focussing on the low energy nodal quasi-particles near the points where the Fermi surface intersects the $k_{x}= \pm k_{y}$ lines. These quasi-particles, which have an energy dispersion corresponding to Dirac fermions $\mathbf{\theta}^{\mathrm{b}}$ in the Meissner phase, are the dominant low-energy excitations which determine the low temperature properties in the superconducting state.

It is possible to derivel the d-wave gap from a model Hamiltonian but the connection of that Hamiltonian with the underlying physics of strongly interacting fermions is unclear. Thus for the purposes of this paper, we assume on phenomenological grounds, the existence of BCS like quasi-particles with an energy gap that has d-wave symmetry. These quasiparticles might not be the real strongly interacting electrons, to whom their connection is unclear at the present time.

Ultrasonic attenuation has historically been a very useful tool in investigating the energies of quasi-particles in conventional superconductors. Early verification $\mathrm{B}$ of the BCS prediction for the temperature dependence of the energy gap was done using this technique. Even afterward 910 this has been a useful tool in the investigation of of superconductivity in heavy fermion superconductors. On the theoretical front calculations of the ultrasonic attenuation in d-wave superconductors have been carried out both in the cleant, 2 , 2,13 and dirty 14,15 limit in the Meissner phase. The former limit which corresponds to $Q l \gg 1$, Q being the ultrasound wave-vector and 1 being the electronic mean free path, is the one considered by us in this paper.

Assuming $100 \mathrm{MHz}$ to be a typical frequency 910 (although ultrasound experiments can be carried out over a large frequency range from $\mathrm{kHz}$ to $\mathrm{GHz}$ ) at which ultrasound experiments are done and taking the sound velocity to be $164 \times 10^{5} \mathrm{~m} / \mathrm{s}$ we find that this translates into the 
requirement $l \gg 4 \mu \mathrm{m}$. This restricts the applicability of our work to the cleanest samples 17 .

While ultrasonic attenuation in the vortex state has recieved attention in the past $18,29,20,21$ the emphasis of earlier workers has been to study effects arising from the coupling of ultrasonic waves to vortex motion. This coupling comes from two different effects: a) The pinning potentials from impurity ions tend to drag the vortices along with them , b) The ionic current due to the ionic displacement will exert a Lorentz force on the vortices. In the clean $\operatorname{limit}(l \geq 4 \mu-m)$ in which we work, the effects of pinning are expected to be weak. The Lorentz force coupling is in any case weak because it is suppressed by a factor of $v / c$ where $v$ is the ionic velocity and $c$ is the velocity of light. We therefore ignore these processes and focus entirely on the phonon damping from the electronic quasi-particles.

In conventional s-wave superconductors, low energy electronic excitations in the vortex state are the bound states localised in the core region of the vortices22. However in d-wave superconductors, because of the presence of nodes in the gap function, the dominant low energy excitations are those in the far region away from the vortex cores. Their contribution is expected to be overwhelmingly larger 23 than that coming from the the cores. Further, experimental studies from STM measurements24.25 reveal that that there are just a few bound states in the vortex cores. Thus we focus exclusively on the excitations in the far region.

To describe the low energy excitations in the mixed phase we make the use of the semiclassical approximation, first discussed 29 in the case of s-wave superconductors. This approximation has been employed for d-wave superconductors in recent times, 23, 26 and its regime of applicability is the range $H_{c 1} \leq H \ll H_{c 2}$, where $\mathrm{H}$ is the applied magnetic field and $H_{c 1}$ and $H_{c 2}$ are the lower critical and upper critical magnetic fields repectively. A striking success of this method has been the prediction 23 of a term linear in the temperature $\mathrm{T}$ whose co-efficient scales as $\sqrt{ } H$ ( $\mathrm{H}$ being the applied magnetic field). This prediction has been verified 27 and has given greater credibility to the semi-classical description. Recently this approximation has been systematized and put on a firm footing by Ramakrishnan and Rajagopal28 who have derived it microscopically.

Using the semi-classical approximation to describe the electronic Green functions in the superconducting state we calculate the imaginary part of the electron density-density correlation function which is proportional to the inverse phonon lifetime. As the sound velocity is only weakly dependent on temperature 16 , the temperature dependence of the attenua- 
tion comes almost entirely from the the inverse lifetime. We find that at low tempeartures, and for parameters appropiate to the cuprates, the ultrasonic attenuation co-efficient in the mixed state has a temperature independent contribution which scales as $\sqrt{H}$. The coefficient of this term and its dependence on the ultrasound wave-vector as well as the leading temperature corrections to it have been explicitly evaluated. These are our main results.

We now present the details of our calculation. The semi-classical approximation can be understood 29 as ignoring, in the first instance, the spatial variation of the supercurrents around a vortex outside its core region. This can be justified on the grounds that the spatial variation of the electronic wave-function has a characteristic length scale $k_{F}^{-1}$ which is much smaller than the smallest length scale associated with vortex currents $\xi$ (the core radius). In the cuprates the parameter $\left(k_{F} \xi\right)^{-1} \simeq 10$ and thus the semi-classical approximation is expected to provide a reasonable description. We then proceed to evaluate the inverse phonon lifetime to first order in $\vec{q}$ where a current has been introduced by taking the energy gap function to be $\Delta_{\vec{q}}\left(\vec{R}_{i}, \vec{R}_{j}\right)=\Delta_{i, j} e^{i \vec{q} \cdot\left(\vec{R}_{i}+\vec{R}_{j}\right)}$. Here $\vec{R}_{i}$ and $\vec{R}_{j}$ are neighbouring sites on a square lattice whose fermions are paired up in a singlet state and $\Delta_{i, j}=\Delta(-\Delta)$ for $\vec{R}_{i}-\vec{R}_{j}$ being in the $\hat{x}(\hat{y})$ direction. The spatial dependence of $\vec{q}=\hat{\phi} / 2 r$ is now restored and the the inverse phonon lifetime is averaged over a unit-cell of the vortex lattice to get our final result for the attenuation co-efficient.

Solving the Bogulibov-deGennes equation for the mean-field d-wave superconductor to linear order in $\vec{q}$ we obtain the results:

$$
G_{\vec{q}}^{\sigma}\left(\vec{R}_{i}-\vec{R}_{j}, i \omega_{n}\right)=\frac{1}{N} \sum_{\vec{k}} e^{i \vec{k} \cdot\left(\vec{R}_{i}-\vec{R}_{j}\right)}\left[\frac{u_{\vec{k}-\vec{q}}^{2}}{i \omega_{n}-E_{\vec{k}-\vec{q}}}+\frac{v_{\vec{q}-\vec{k}}^{2}}{i \omega_{n}+E_{\vec{q}-\vec{k}}}\right]
$$

where $u_{\vec{k}}^{2}=\left(1+\epsilon_{\vec{k}} / E_{\vec{k}}^{0}\right) / 2, v_{\vec{k}}^{2}=\left(1-\epsilon_{\vec{k}} / E_{\vec{k}}^{0}\right) / 2, \epsilon_{\vec{k}}=\xi_{\vec{k}}-\mu, \xi_{\vec{k}}$ being the band energy and $\mu$ the chemical potential, $E_{\vec{k}}^{0}=\sqrt{\epsilon_{\vec{k}}^{2}+\Delta_{\vec{k}}^{2}}, \Delta_{\vec{k}}=\Delta\left(\cos k_{x} a-\cos k_{y} a\right)$ being the d-wave gap and $E_{\vec{k}}=E_{\vec{k}}^{0}+\vec{q} \cdot \nabla_{k} \xi_{\vec{k}}$ being the Doppler shifted quasi-particle energy. Here

$$
G_{\vec{q}}^{\sigma}\left(\vec{R}_{i}-\vec{R}_{j}, i \omega_{n}\right)=-\int_{0}^{\beta} d \tau<c_{i, \sigma}(\tau) c_{j, \sigma}^{\dagger}(0)>e^{i \omega_{n} \tau}
$$

is the "normal" Green function. Similarly the anomalous Green functions are given by:

$$
F_{\vec{q}}\left(\vec{R}_{i}, \vec{R}_{j}, i \omega_{n}\right)=\frac{e^{i \vec{q} \cdot\left(\overrightarrow{R_{i}}+\vec{R}_{j}\right)}}{N} \sum_{\vec{k}} u_{\vec{k}} v_{\vec{k}}\left(\frac{e^{-i \vec{k} \cdot\left(\overrightarrow{R_{i}}-\overrightarrow{R_{j}}\right)}}{i \omega_{n}-E_{\vec{k}}}-\frac{e^{i \vec{k} \cdot\left(\overrightarrow{R_{i}}-\overrightarrow{R_{j}}\right)}}{i \omega_{n}+E_{\vec{k}}}\right)
$$

and 


$$
F_{\vec{q}}^{+}\left(\vec{R}_{i}, \vec{R}_{j}, i \omega_{n}\right)=\frac{e^{-i \vec{q} \cdot\left(\vec{R}_{i}+\vec{R}_{j}\right)}}{N} \sum_{\vec{k}} u_{\vec{k}} v_{\vec{k}}\left(\frac{e^{-i \vec{k} \cdot\left(\vec{R}_{i}-\vec{R}_{j}\right)}}{i \omega_{n}-E_{\vec{k}}}-\frac{e^{i \vec{k} \cdot\left(\vec{R}_{i}-\vec{R}_{j}\right)}}{i \omega_{n}+E_{\vec{k}}}\right)
$$

Here $u_{\vec{k}} v_{\vec{k}}=\Delta_{\vec{k}} / 2 E_{\vec{k}}^{0}$ and the Green functions $F_{\vec{q}}$ and $F_{\vec{q}}^{+}$are defined as

$$
\begin{aligned}
& F_{\vec{q}}\left(\vec{R}_{i}, \vec{R}_{j}, i \omega_{n}\right)=-\int_{0}^{\beta} d \tau<c_{i, \downarrow}(\tau) c_{j, \uparrow}(0)>e^{i \omega_{n} \tau} \\
& F_{\vec{q}}^{+}\left(\vec{R}_{i}, \vec{R}_{j}, i \omega_{n}\right)=-\int_{0}^{\beta} d \tau<c_{i, \uparrow}^{\dagger}(\tau) c_{j, \downarrow}^{\dagger}(0)>e^{i \omega_{n} \tau}
\end{aligned}
$$

Using the Green functions in equations (1),(3) and (4) we compute the imaginary part of the density-density correlation function which is given by

$$
\chi_{\vec{q}}^{\prime \prime}(\vec{Q}, \omega)=X_{1}+X_{2}+X_{3}+X_{4}
$$

where

$$
\begin{gathered}
X 1=\frac{-2 \pi}{N} \sum_{\vec{k}}\left[n\left(E_{\vec{k}}\right)-n\left(E_{\vec{k}+\vec{Q}}\right)\right]\left(u_{\vec{k}+\vec{Q}}^{2} u_{\vec{k}}^{2}-\frac{\Delta_{\vec{k}} \Delta_{\vec{k}+\vec{Q}}}{4 E_{\vec{k}}^{0} E_{\vec{k}+\vec{Q}}^{0}}\right) \delta\left(\omega+E_{\vec{k}}-E_{\vec{k}+\vec{Q}}\right) \\
X 2=\frac{-2 \pi}{N} \sum_{\vec{k}}\left[n\left(E_{-\vec{k}}\right)-n\left(E_{-\vec{k}-\vec{Q}}\right)\right]\left(v_{\vec{k}+\vec{Q}}^{2} v_{\vec{k}}^{2}-\frac{\Delta_{\vec{k}} \Delta_{\vec{k}+\vec{Q}}}{4 E_{\vec{k}}^{0} E_{\vec{k}+\vec{Q}}^{0}}\right) \delta\left(\omega-E_{-\vec{k}}+E_{-\vec{k}-\vec{Q}}\right) \\
X 3=\frac{-2 \pi}{N} \sum_{\vec{k}}\left[1-n\left(E_{-\vec{k}}\right)-n\left(E_{\vec{k}+\vec{Q}}\right)\right]\left(u_{\vec{k}+\vec{Q}}^{2} v_{\vec{k}}^{2}+\frac{\Delta_{\vec{k}} \Delta_{\vec{k}+\vec{Q}}}{4 E_{\vec{k}}^{0} E_{\vec{k}+\vec{Q}}^{0}}\right) \delta\left(\omega-E_{-\vec{k}}-E_{\vec{k}+\vec{Q}}\right),
\end{gathered}
$$

and

$$
X 4=\frac{2 \pi}{N} \sum_{\vec{k}}\left[1-n\left(E_{\vec{k}}\right)-n\left(E_{-\vec{k}-\vec{Q}}\right)\right]\left(v_{\vec{k}+\vec{Q}}^{2} u_{\vec{k}}^{2}+\frac{\Delta_{\vec{k}} \Delta_{\vec{k}+\vec{Q}}}{4 E_{\vec{k}}^{0} E_{\vec{k}+\vec{Q}}^{0}}\right) \delta\left(\omega+E_{\vec{k}}+E_{-\vec{k}-\vec{Q}}\right) .
$$

In s-wave superconductors the contribution of $X_{3}$ and $X_{4}$ are zero as the ultrasound frequency $\omega \ll 2 \Delta$ and so the $\delta$-fn condition in Eqs.(10) and (11) can never be satisfied. In the d-wave case the presence of nodes in $\Delta_{\vec{k}}$ means that $X_{3}$ and $X_{4}$ will be finite. However, as the phase space for them is limited, their contribution is expected to be small and so we focus exclusively on $X_{1}$ and $X_{2}$ which make the dominant contribution to $\chi_{\vec{q}}^{\prime \prime}(\vec{Q}, \omega)$.

Now expanding to the leading order in $\omega$ we find 


$$
\begin{array}{r}
\chi_{\vec{q}}^{\prime \prime}(\vec{Q}, \omega)=\frac{2 \pi \omega}{N} \sum_{\vec{k}}\left[n^{\prime}\left(E_{\vec{k}}\right) \delta\left(E_{\vec{k}}-E_{\vec{k}+\vec{Q}}\right)\left(u_{\vec{k}+\vec{Q}}^{2} u_{\vec{k}}^{2}-\frac{\Delta_{\vec{k}} \Delta_{\vec{k}+\vec{Q}}}{4 E_{\vec{k}}^{0} E_{\vec{k}+\vec{Q}}^{0}}\right)+n^{\prime}\left(E_{-\vec{k}}\right)\right. \\
\left.\delta\left(E_{-\vec{k}}-E_{-\vec{k}-\vec{Q}}\right)\left(v_{\vec{k}+\vec{Q}}^{2} v_{\vec{k}}^{2}-\frac{\Delta_{\vec{k}} \Delta_{\vec{k}+\vec{Q}}}{4 E_{\vec{k}}^{0} E_{\vec{k}+\vec{Q}}^{0}}\right)\right]
\end{array}
$$

We next expand $\chi$ " to leading order in the ultrasound wave-vector $\vec{Q}$ and on making the substitution $\vec{k} \rightarrow \overrightarrow{-k}$ in the second term in Eq. (12) we arrive at the result

$$
\chi_{\vec{q}}^{\prime \prime}(\vec{Q}, \omega)=\frac{2 \pi \omega}{N} \Sigma_{\vec{k}} n^{\prime}\left(E_{\vec{k}}^{0}+\vec{q} \cdot \vec{\nabla}_{k} \xi_{\vec{k}}\right) \frac{\epsilon_{\vec{k}}^{2}}{E_{\vec{k}}^{0^{2}}} \delta\left(\vec{Q} \cdot \vec{\nabla}_{k} E_{\vec{k}}^{0}+q_{\alpha} Q_{\beta} \frac{\partial^{2} \xi_{\vec{k}}}{\partial k_{\alpha} \partial k_{\beta}}\right)
$$

where a sum over repeated indices is implied in the arguement of the $\delta$ function.

To do the $\vec{k}$ summation in Eq. (13) we only consider $\theta^{6}$ the fermions near the nodes. This approximation is reasonable at low temperatures $\left(k_{B} T \ll \Delta\right)$. For the node near $\vec{P}_{a}=(\pi / 2 a, \pi / 2 a)$ upon linearising the band energies and gap function, we have: $\xi_{\vec{k}} \simeq v_{F} k_{1}$ and $\Delta_{\vec{k}} \simeq v_{\Delta} k_{2}$ where $k_{1}$ and $k_{2}$ are co-ordinates normal and tangential respectively to the Fermi surface at the node. In terms of the co-ordinates $k_{1}$ and $k_{2}$ we find that the contribution from this node to $\chi_{\vec{q}}^{\prime(a)}$ can be written as

$$
\begin{array}{r}
\chi_{\vec{q}}^{\prime(a)}(\vec{Q}, \omega) \simeq \frac{a^{2} \omega}{2 \pi} \int d k_{1} \int d k_{2} n^{\prime}\left[\sqrt{v_{F}^{2} k_{1}^{2}+v_{\Delta}^{2} k_{2}^{2}}+\rho_{0}\right] \frac{v_{F}^{2} k_{1}^{2}}{v_{F}^{2} k_{1}^{2}+v_{\Delta}^{2} k_{2}^{2}} \\
\delta\left[\frac{\alpha v_{F} k_{1}+\beta v_{\Delta} k_{2}}{\sqrt{v_{F}^{2} k_{1}^{2}+v_{\Delta}^{2} k_{2}^{2}}}-\alpha_{1} v_{F} k_{1}-\alpha_{2} v_{F} k_{2}\right]
\end{array}
$$

Here $\alpha=v_{F} Q \cos (\pi / 4-\theta), \beta=v_{\Delta} Q \sin (\pi / 4-\theta), \alpha_{1}=\left(Q q a^{2} / 2\right) \cos (\psi-\theta), \alpha_{2}=$ $-\left(Q q a^{2} / 2\right) \cos (\psi+\theta), \rho_{0}=q v_{F} \cos (\psi-\pi / 4)$ and $\theta$ and $\psi$ are the angles made by $\vec{Q}$ and $\vec{q}$ respectively with respect to the $k_{1}$ axis. We now introduce the polar co-ordinates $v_{F} k_{1}=$ $\rho \cos \phi$ and $v_{\Delta} k_{2}=\rho \sin \phi$. Then upon performing the $\phi$ integral we find $\chi_{\vec{q}}^{\prime \prime}(a)$ reduces to

$$
\chi_{\vec{q}}^{\prime \prime(a)}(\vec{Q}, \omega)=\frac{a^{2} \omega}{\pi v_{F} v_{\Delta}} \int_{0}^{\rho_{c}} d \rho \rho n^{\prime}\left[\rho+\rho_{0}\right] f_{a}[\rho]
$$

where $f_{a}[\rho]=\frac{B^{2}}{\left(A^{2}+B^{2}\right)^{3 / 2}}$ and $A=\alpha-\alpha_{1} \rho, B=\beta-\left(v_{F} / v_{\Delta}\right) \alpha_{2} \rho$ and $\rho_{c}=\sqrt{\pi v_{F} v_{\Delta}} / a$ is a cutoff introduced to preserve the volume of the Brillouin zone while doing the $\vec{k}$-integration.

Introducing the variable $\rho^{\prime}=\rho+\rho_{0}$ we have $\chi^{\prime a}=\chi^{\prime \prime(a 1)}+\chi^{\prime(a 2)}$ where

$$
\chi_{\vec{q}}^{\prime \prime(a 1)}(\vec{Q}, \omega)=\frac{a^{2} \omega}{\pi v_{F} v_{\Delta}} \int_{\rho_{0}}^{\rho_{c}} d \rho^{\prime} \rho^{\prime} n^{\prime}\left[\rho^{\prime}\right] f_{a}\left[\rho^{\prime}-\rho_{0}\right]
$$


and

$$
\chi_{\vec{q}}^{\prime \prime(a 2)}(\vec{Q}, \omega)=-\frac{a^{2} \omega}{\pi v_{F} v_{\Delta}} \rho_{0} \int_{\rho_{0}}^{\rho_{c}} d \rho^{\prime} n^{\prime}\left[\rho^{\prime}\right] f_{a}\left[\rho^{\prime}-\rho_{0}\right]
$$

In writing Eqs. (16) and (17) we have made use of $\rho_{c} \gg \rho_{0}$ which follows from using $q_{\max }=1 / 2 \xi$ and $\xi \approx 30 \AA, v_{F} / a \approx 192 \mathrm{meV}$ and $v_{\Delta} / a \approx 28 \mathrm{meV}$ for parameters appropriate to the cuprates 6 .

In an analogous fashion we evaluate the corresponding contributions to $\chi_{\vec{q}}^{\prime \prime}$ from the nodes near $\overrightarrow{P_{b}}=(-\pi / 2 a,-\pi / 2 a), \vec{P}_{c}=(-\pi / 2 a, \pi / 2 a)$ and $\vec{P}_{d}=(\pi / 2 a,-\pi / 2 a)$. We first focus on the type of terms in Eq. (16). In this case we find, on expanding to linear order in $q$, that the contribution linear in $q$ exactly vanishes due to cancellations from the terms coming from different nodes. We thus find $\chi_{\vec{q}}^{\prime \prime(1)}=\chi_{\vec{q}}^{\prime \prime(a 1)}+\chi_{\vec{q}}^{\prime(b 1)}+\chi_{\vec{q}}^{\prime \prime(c 1)}+\chi_{\vec{q}}^{\prime \prime(d 1)}$ to be given by

$$
\chi_{\vec{q}}^{\prime(1)}=-\frac{2 \ln 2 \omega a^{2} k_{B} T}{\pi v_{F} v_{\Delta}}\left[\frac{\beta^{2}}{\left(\alpha^{2}+\beta^{2}\right)^{3 / 2}}+\frac{\eta^{2}}{\left(\lambda^{2}+\eta^{2}\right)^{3 / 2}}\right]
$$

to linear order in $\vec{q}$. Once again we have used $k_{B} T \ll q v_{F} \ll \rho_{c}$. Here $\alpha$ and $\beta$ are previously defined and we have introduced the parameters $\lambda=v_{F} Q \sin (\pi / 4-\theta), \eta=$ $v_{\Delta} Q \cos (\pi / 4-\theta)$. The result obtained for this term is identical to the zero field result for the attenuation previously calculated in Ref. (12). We next turn our attention to the terms of the type written in Eq.(17). Here on adding the contributions from the nodes near $\vec{P}_{a}$ and $\vec{P}_{b}$, we find to leading order in $q$,

$$
\chi_{\vec{q}}^{\prime \prime(a 2)}+\chi_{\vec{q}}^{\prime(b 2)}=-\frac{\omega a^{2} q}{\pi v_{\Delta}} \cos (\psi-\pi / 4) \frac{\beta^{2}}{\left(\alpha^{2}+\beta^{2}\right)^{3 / 2}} \tanh \left(\frac{q v_{F} \cos (\psi-\pi / 4)}{2 k_{B} T}\right)
$$

Similarly the nodes near $\vec{P}_{c}$ and $\vec{P}_{d}$ yield the contribution

$$
\chi_{\vec{q}}^{\prime \prime(c 2)}+\chi_{\vec{q}}^{\prime \prime(d 2)}=-\left(\frac{\omega a^{2} q}{\pi v_{\Delta}}\right) \cos (\psi+\pi / 4) \frac{\eta^{2}}{\left(\lambda^{2}+\eta^{2}\right)^{3 / 2}} \tanh \left(\frac{q v_{F} \cos (\psi+\pi / 4)}{2 k_{B} T}\right)
$$

It is straightforward to see that for $T \rightarrow 0$, Eqs. (19) and (20) reduce to

$$
\chi_{\vec{q}}^{\prime \prime(a 2)}+\chi_{\vec{q}}^{\prime(b 2)}=-\left(\frac{\omega a^{2} q}{\pi v_{\Delta}}\right) \cos (\psi-\pi / 4) \frac{\beta^{2}}{\left(\alpha^{2}+\beta^{2}\right)^{3 / 2}}[\theta(\cos (\psi-\pi / 4))-\theta(-\cos (\psi-\pi / 4))]
$$

and

$$
\chi_{\vec{q}}^{\prime \prime(c 2)}+\chi_{\vec{q}}^{\prime \prime(d 2)}=-\left(\frac{\omega a^{2} q}{\pi v_{\Delta}}\right) \cos (\psi+\pi / 4) \frac{\eta^{2}}{\left(\lambda^{2}+\eta^{2}\right)^{3 / 2}}[\theta(\cos (\psi+\pi / 4))-\theta(-\cos (\psi+\pi / 4))]
$$

Eqs.(21) and (22) are also obtained directly by taking the $\mathrm{T}=0$ limit for n' in Eq.(15) and its analogues for the other 3 nodes. 
To proceed further we now restore $\vec{q}=\hat{\phi} / 2 r$ corresponding to the supercurrents around a vortex where the vector potential has been ignored as the Ginzburg-Landau parameter $\kappa \gg 1$. Assuming a vortex lattice with circular unit-cells we find that the radius of the cells $R_{c} \sim \xi \sqrt{H_{c 2} / H}$. We then average $\chi_{\vec{q}}^{\prime \prime}$ over one unit cell to obtain

$$
\chi^{\prime \prime}(H, T)=\frac{\int_{\xi}^{R_{c}} r d r \int_{0}^{2 \pi} d \psi \chi_{\vec{q}}^{\prime \prime}}{\int_{\xi}^{R_{c}} r d r \int_{0}^{2 \pi} d \psi}
$$

to obtain our final result for $\chi "$.

In order to perform the averaging in Eq. (23) we make the approximation $\tanh (x) \simeq x$ for $|x|<1, \tanh (x) \simeq 1$ for $x>1$ and $\tanh (x) \simeq-1$ for $x<-1$. This approximation interpolates between the asymptotically exact behaviour at small and large $|x|$. Further it reproduces the exact result that can be directly calculated for $T=0$. In that case the integral in Eq. (23) is elementary and can be done by substituting the expressions in Eqs. (21) and (22). We then obtain to leading order in the small parameter $\left(\xi k_{B} T / v_{F}\right)^{2}\left(H_{c 2} / H\right)$ :

$$
\chi^{\prime \prime(2)}(H, T)=-\frac{2 \omega a^{2}}{\pi^{2} v_{\Delta} \xi}\left(\frac{H}{H_{c 2}}\right)^{1 / 2}\left[1-\frac{8 \xi^{2}}{9}\left(\frac{k_{B} T}{v_{F}}\right)^{2} \frac{H_{c 2}}{H}\right]\left[\frac{\beta^{2}}{\left(\alpha^{2}+\beta^{2}\right)^{3 / 2}}+\frac{\eta^{2}}{\left(\lambda^{2}+\eta^{2}\right)^{3 / 2}}\right]
$$

The assumption about the small parameter implies that the regime of validity of our result is for $k_{B} T \ll \frac{v_{F}}{\xi}\left(\frac{H}{H_{c 2}}\right)^{1 / 2}$ together with the condition $H_{c 1} \leq H \ll H_{c 2}$. For parameters relevant to the cuprates $H_{c 2} / H_{c 1} \simeq 100$ at low temperatures. This restricts the temperature window to $T \ll 1 K$ at $H=H_{c 1}$. Our result for a temperature independent contribution to the attenuation that scales as $\sqrt{H}$ is entirely understandable as it has its origin in a finite density of states at the Fermi-energy 23 whose size is proportional to $\sqrt{H}$ and whose signature is seen in the specific heat measurements27.

The actual ultrasonic attenuation coefficient is related to $\chi$ " by

$$
\alpha_{S}(T, H)=M(\vec{Q}) \chi ”(\vec{Q}, T, H)
$$

where $M(\vec{Q})$ is a constant which depends on the sound velocity and the electron-phonon matrix element and the ultrasound frequency. Then on combining Eqs. (18) and (24) and considering the fact that Eq. (18) contains the result for the Meissner phase $(H=0)$ we obtain the result:

$$
\frac{\alpha_{S}(T, H)-\alpha_{S}(T, H=0)}{\alpha_{S}(T, H=0)}=\frac{v_{F}}{\pi \xi}\left(\frac{H}{H_{c 2}}\right)^{1 / 2} \frac{1}{\ln 2 k_{B} T}\left[1-\frac{8 \xi^{2}}{9}\left(\frac{k_{B} T}{v_{F}}\right)^{2} \frac{H_{c 2}}{H}\right]
$$


whose window of validity has been described above. This result is remarkable because it is

independent of the ultrasound wave-vector $\vec{Q}$ as well as the gap increase parameter $v_{\Delta}$. Thus a measurement of the ultrasonic attenuation at low temperatures and at fields above $H_{c 1}$ would enable a determination of any one of the parameters $v_{F}, \xi$ and $H_{c 2}$ given a knowledge of the other two.

We now discuss some shortcomings of our work. We have assumed perfectly welldefined quasi-particles thus ignoring the incoherent spectral weight seen in photoemission experiments. The semi-classical approximation used by us may not accurately describe all the physical effects due to the scattering of quasi-particles from the supercurrents in the vortex state. Our results are restricted to the clean limit and are applicable only to very clean samples.

Finally we conclude by recapitulating the main points of this paper. We have employed the semi-classical approximation to evaluate the phonon damping due to the electronic quasi-particles in a d-wave superconductor. We find that for parameters appropiate to the cuprates, in a temperature window $k_{B} T \ll 1 K$, there is a temperature independent contribution to the ultrasonic attenuation whose magnitude scales as $\sqrt{H}$.

\section{Acknowledgments}

One of us (DMG) thanks T.V.Ramakrishnan for useful comments.

1 J. G. Bednorz and K. A. Muller, Z. Phys.B64, 189 (1986).

2 P. W. Anderson, The Theory of Superconductivity in High-Tc Cuprates, (Princeton University Press, Princeton, 1997).

3 M. Randeria et. al, Phys. Rev. Lett. 74, 4951 (1995).

4 J.F.Annett, N.Goldenfeld and A.J.Leggett, in Physical Properties of High Temperature Superconductors, Vol-5, ed. D.M.Ginsberg (World Scientific, Singapore,1996), p. 375.

5 C. C. Tsuei and J. R. Kirtley, Rev. Mod. Phys. 72, 969 (2000).

6 P.A.Lee and X.G.Wen, Phys. Rev. Lett. 78, 4111 (1997).

7 T.Gupta and D.M.Gaitonde, Mod.Phys.Lett. B15, 269 (2001). 
8 R. W. Morse in Progress in Cryogenics, Vol-I, ed. K. Mendelssohn (Heywood, London, 1959) p.219.

9 D. J. Bishop et. al., Phys. Rev. Lett., 53, 1009 (1984).

10 B. Batlogg et. al., Phys. Rev. Lett., 55, 1319 (1985).

11 S.N. Coppersmith and R.A. Klemm, Phys. Rev. Lett.56, 1870(1986).

12 I.Vekhter, E.J. Nicol and J.P. Carbotte, Phys. Rev. B59, 7123 (1999).

13 W.C.Wu and J.P.Carbotte, Phys. Rev. B60, 14943 (1999).

14 J.Moreno and P.Coleman, Phys. Rev. B53, R2995 (1996).

15 M.B.Walker, M.F.Smith and K.V.Samokhin cond mat/0105109 (unpublished)

16 S. Bhattacharya et al, Phys. Rev. B37, 5901 (1988).

17 A. Hosseini et. al., cond-mat/9811041 (unpublished).

18 J. Pankert et. al., Phys. Rev. Lett.65, 3052 (1990).

19 D. Dominguez et. al., Phys. Rev. Lett.74, 2579 (1995).

20 D. Dominguez et. al., Phys. Rev.B53, 6682 (1996).

21 G. Blatter and B. Ivlev, Phys. Rev.B52, 4588 (1995).

22 C. Caroli, P. G. de Gennes and J. Matricon, Phys. Lett.9, 307 (1964).

23 G. E. Volovik, JETP Lett. 58, 469 (1993).

24 I. Maggio-Aprile et. al., Phys. Rev. Lett. 75, 2574 (1995).

25 Ch. Renner et. al., Phys. Rev. Lett. 80, 3606 (1998).

26 C. Kubert and P. Hirschfeld, Solid State Comm.105, 459 (1998).

27 K. Moller et. al., Phys. Rev. Lett.73, 2744 (1994).

28 T. V. Ramakrishnan and A. K. Rajagopal, Jour. of Stat. Phys.103, 441 (2001).

29 P. G. de Gennes Superconductivity of metals and alloys (Addison-Wesley, Reading, MA 1989) 